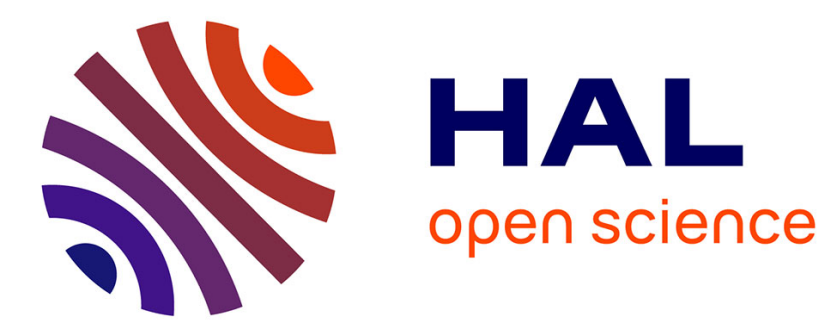

\title{
Decay estimates to equilibrium for some asymptotically autonomous semilinear evolution equations
}

Imen Ben Hassen

\section{To cite this version:}

Imen Ben Hassen. Decay estimates to equilibrium for some asymptotically autonomous semilinear evolution equations. 2009. hal-00392110

\section{HAL Id: hal-00392110 \\ https://hal.science/hal-00392110}

Preprint submitted on 5 Jun 2009

HAL is a multi-disciplinary open access archive for the deposit and dissemination of scientific research documents, whether they are published or not. The documents may come from teaching and research institutions in France or abroad, or from public or private research centers.
L'archive ouverte pluridisciplinaire HAL, est destinée au dépôt et à la diffusion de documents scientifiques de niveau recherche, publiés ou non, émanant des établissements d'enseignement et de recherche français ou étrangers, des laboratoires publics ou privés. 


\title{
Decay estimates to equilibrium for some asymptotically autonomous semilinear evolution equations
}

\author{
Imen Ben Hassen* \\ Département de Mathématiques \\ Faculté des Sciences de Bizerte \\ 7021 Jarzouna, Bizerte, Tunisia \\ imen_benhassen@yahoo.fr
}

Abstract. We estimate the rate of decay of the difference between a solution and its limiting equilibrium for the nonautonomous first order problem

$$
\dot{u}+\mathcal{M} u=g(t), \quad t \in \mathbb{R}_{+},
$$

and for the second order problem

$$
\ddot{u}+\dot{u}+\mathcal{M} u=g(t), \quad t \in \mathbb{R}_{+},
$$

where $g$ is a function which tends to 0 when $t$ tends to $\infty$.

Keywords : convergence, rate of decay, analytic nonlinearity, nonautonomous, evolution equation.

*The author gratefully acknowledges support by the Coopération franco-tunisienne dans le cadre de l'accord CNRS/DGRSRT (No. 21817): Systèmes dynamiques et équations d'évolution 


\section{Introduction}

In this paper we estimate the rate of convergence to equilibrium of bounded solutions of asymptotically autonomous semilinear evolution equations such as for example the heat equation

$$
u_{t}-\Delta u+f(u)=g(t, x)
$$

or the wave equation

$$
u_{t t}+u_{t}-\Delta u+f(u)=g(t, x) .
$$

In the autonomous case, i.e. $g=0$, many authors have studied the asymptotic behavior of solutions for both equations. They used different assumptions on the nonlinearity $f$ and the domain $\Omega,[17],[14],[15],[12],[3],[8],[1],[10],[11],[6]$, so as to prove convergence to equilibrium. The basic argument used by L. Simon [15] in order to get his convergence result for solutions of equation (1), is the Lojasiewicz inequality [13] for real analytic functions defined on $\mathbb{R}^{d}$ which he generalized to the infinite dimensional case. This inequality is called Lojasiewicz-Simon inequality. The same result has been proved for equation (2) under the same assumptions [10]. The decay estimates of solutions of both equations have been studied by A. Haraux and M.A. Jendoubi. They established in [5] the rate of convergence to equilibrium of such solutions under relevant growth conditions on $f$.

In the nonautonomous case, i.e. $g \neq 0$, recently Huang and Takač [9] have proved convergence to equilibrium of bounded solutions of equation (1) under the assumption that $f$ is analytic and $g$ is such that

$$
\sup _{t \in \mathbb{R}_{+}} t^{1+\delta} \int_{t}^{\infty}\|g(s)\|_{L^{2}(\Omega)}^{2} d s<\infty
$$

for some $\delta>0$.

Under these assumptions, R. Chill and M.A. Jendoubi [2] have proved the same result for equation (2).

The main objective of this article is to study the rate of decay of solutions of equations (1) and (2).

Our article is organized as follows : In Section 2, we present the first order and second order abstract equations which we will study and we state the main results. Sections 3 and 4 are devoted to the proofs of the main results. In Section 5 , we give some examples of applications of our abstract results. Among these are the finite dimensional gradient systems and equations (1) and (2). 


\section{Main results}

Throughout this article we let $H$ and $V$ be two Hilbert spaces. We assume that $V$ is densely and continuously embedded into $H$. Identifying $H$ with its dual $H^{\prime}$, we obtain $V \hookrightarrow H=H^{\prime} \hookrightarrow V^{\prime}$. We denote by $\langle\cdot, \cdot\rangle$ scalar products and duality relations; the spaces in question will be specified by subscripts. Throughout, we let $C_{1} \geq 0$ be such that

$$
\|v\|_{V^{\prime}} \leq C_{1}\|v\|_{H} \leq C_{1}^{2}\|v\|_{V}, \quad v \in V .
$$

Other constants in the calculations will be denoted by $C_{i}(i \geq 2)$.

Let $E \in C^{1}(V, \mathbb{R})$, and denote by $\mathcal{M} \in C\left(V, V^{\prime}\right)$ the first derivative of $E$. Let furthermore $g \in L_{\text {loc }}^{1}\left(\mathbb{R}_{+} ; H\right)$.

We study the following two abstract Cauchy problems: the first order problem

$$
\begin{cases}\dot{u}(t)+\mathcal{M}(u(t))=g(t), & t \geq 0, \\ u(0)=u_{0}, & u_{0} \in H,\end{cases}
$$

and the second order problem

$$
\begin{cases}\ddot{u}(t)+\dot{u}(t)+\mathcal{M}(u(t))=g(t), & t \geq 0, \\ u(0)=u_{0}, & u_{0} \in V, \\ \dot{u}(0)=u_{1}, & u_{1} \in H .\end{cases}
$$

A function $u: \mathbb{R}_{+} \rightarrow V$ will be called a solution of equation (4), if $u \in W_{\text {loc }}^{1,1}\left(\mathbb{R}_{+} ; V^{\prime}\right) \cap$ $L_{l o c}^{1}\left(\mathbb{R}_{+} ; V\right), u(0)=u_{0}$, and if $u$ satisfies equation (4). Similarly, a function $u: \mathbb{R}_{+} \rightarrow V$ is called a solution of equation (5), if $u \in W_{l o c}^{2,1}\left(\mathbb{R}_{+} ; V^{\prime}\right) \cap L_{l o c}^{1}\left(\mathbb{R}_{+} ; V\right), u(0)=u_{0}, \dot{u}(0)=u_{1}$, and if $u$ satisfies equation (5).

We define the $\omega$-limit set of a solution $u$ of (4) or (5) by

$$
\omega(u)=\left\{\varphi \in V: \exists t_{n} \rightarrow+\infty \text { such that } \lim _{n \rightarrow \infty}\left\|u\left(t_{n}\right)-\varphi\right\|_{V}=0\right\} .
$$

If $u: \mathbb{R}_{+} \rightarrow V$ is a solution of equation (4) (resp. equation (5)) such that the range $\{u(t): t \geq 1\}$ is relatively compact in $V$, then the $\omega$-limit set $\omega(u)$ is nonempty and it has been shown by R. Chill and M.A. Jendoubi [2] that with an additional geometric condition on the function $E$ and its derivative $\mathcal{M}$ the $\omega$-limit set is reduced to one point, and that the solution converges.

Definition 2.1 We say that the function E satisfies the Lojasiewicz-Simon inequality near some point $\varphi \in V$, if there exist constants $\theta \in\left(0, \frac{1}{2}\right], \eta>0$ and $\sigma>0$ such that :

$$
\text { for all } v \in V \text { with }\|v-\varphi\|_{V} \leq \sigma,|E(v)-E(\varphi)|^{1-\theta} \leq \eta\|\mathcal{M}(v)\|_{V^{\prime}} .
$$

The constant $\theta$ is called the Lojasiewicz exponent. 
Our main results read as follows.

Theorem 2.2 Let $u: \mathbb{R}_{+} \rightarrow V$ be a solution of equation (4), and assume that

1. $u \in W_{\text {loc }}^{1,1}\left(\mathbb{R}_{+} ; V\right) \cap W_{\text {loc }}^{1,2}\left(\mathbb{R}_{+} ; H\right)$.

2. The set $\{u(t): t \geq 1\}$ is relatively compact in $V$.

3. There exists $\varphi \in \omega(u)$ such that $E$ satisfies the Lojasiewicz-Simon inequality with exponent $\theta$ near $\varphi$.

4. There exist constants $c>0$ and $\delta>0$ such that

$$
\int_{t}^{\infty}\|g(s)\|_{H}^{2} d s \leq \frac{c}{(1+t)^{1+\delta}}
$$

Then there exists a constant $C>0$ such that for all $t \geq 0$ we have

$$
\|u(t)-\varphi\|_{H} \leq C(1+t)^{-\beta}, \text { where } \beta=\inf \left\{\frac{\theta}{1-2 \theta}, \frac{\delta}{2}\right\} .
$$

Theorem 2.3 Let $u: \mathbb{R}_{+} \rightarrow V$ be a solution of equation (5), and assume that

1. $u \in W_{l o c}^{1,2}\left(\mathbb{R}_{+} ; V\right) \cap W_{l o c}^{2,2}\left(\mathbb{R}_{+} ; H\right)$.

2. The set $\{(u(t), \dot{u}(t)): t \geq 1\}$ is relatively compact in $V \times H$.

3. $E \in C^{2}(V ; \mathbb{R})$.

4. If $K: V^{\prime} \rightarrow V$ denotes the duality map, then the operator $K \circ \mathcal{M}^{\prime}(v) \in \mathcal{L}(V)$ extends to a bounded linear operator on $H$ for every $v \in V$, and $K \circ \mathcal{M}^{\prime}: V \rightarrow \mathcal{L}(H)$ maps bounded sets into bounded sets.

5. There exists $\varphi \in \omega(u)$ such that $E$ satisfies the Lojasiewicz-Simon inequality with exponent $\theta$ near $\varphi$.

6. There exist constants $c>0$ and $\delta>0$ such that (7) holds.

Then there exists a constant $C^{\prime}$ such that for all $t \geq 0$ we have

$$
\|u(t)-\varphi\|_{H} \leq C^{\prime}(1+t)^{-\beta} \text {, where } \beta \text { is as in }(8) \text {. }
$$


Remarks 2.4 (a) Note that if $g=0$ and $\theta=\frac{1}{2}$, A. Haraux and M.A. Jendoubi showed in [5] exponential decay of solutions for both equations (1) and (2).

(b) In theorems 2.2 and 2.3 , if $\theta=\frac{1}{2}$ then $\beta=\frac{\delta}{2}$.

(c) Note that if $\theta=\frac{1}{2}$ and we have instead of the condition (7),

$$
\int_{t}^{\infty}\|g(s)\|_{H}^{2} d s \leq C \exp (-\delta t)
$$

where $C$ and $\delta$ are two positive constants, we can show an exponential decay of solutions for both equations (1) and (2).

(d) Note that if $\|g(s)\|_{H} \leq \frac{c}{(1+s)^{1+\frac{\delta}{2}}}$, where $c$ is a positive constant, then condition (7) is verified.

(e) Condition (7) implies in turn that $\int_{t}^{\infty}\|g(s)\|_{H} d s \leq \frac{c^{\prime}}{(1+t)^{\frac{\delta}{2}}}$, where $c^{\prime}$ is a positive constant. Moreover if we consider the case $V=H=\mathbb{R}, \mathcal{M}=0$ and $g(t) \simeq K^{\prime} t^{-\lambda}$, where $K^{\prime}$ is a constant, the existence of a solution with $\left\|u(t)-u_{\infty}\right\| \leq c^{\prime \prime} t^{-\frac{\delta}{2}}$, where $c^{\prime \prime}$ is a positive constant, is equivalent to the assertion $\lambda \geq \frac{\delta}{2}+1$. This explains why the power $\frac{\delta}{2}$ appears in the calculations.

Remarks 2.5 The estimates obtained in this paper are optimal. Here are some examples which show the optimality of our results even when $V=H=\mathbb{R}$ :

(a) let consider the case $\frac{\theta}{1-2 \theta} \geq \frac{\delta}{2}$, then by choosing $E=0$ and $g(t)=t^{-\left(1+\frac{\delta}{2}\right)}$ it is easy to check that estimate (8) is optimal.

Now if we consider the case $\frac{\theta}{1-2 \theta}<\frac{\delta}{2}$, then by choosing $E(u)=|u|^{\frac{1}{\theta}}$, where $\theta<\frac{1}{2}$ and $g=0$, we can easily see that estimate (8) is also optimal in this case.

(b) It is not difficult using [4] to see that the estimate (8) is also optimal in theorem 2.3.

In the proof of our theorems we shall establish some differential inequalities. The following lemma will allow us to deduce from those inequalities the desired decay estimates.

Lemma 2.6 Let $\phi \in W_{\text {loc }}^{1,1}\left(\mathbb{R}_{+}, \mathbb{R}_{+}\right)$. We suppose that there exists constants $K_{1}>0, K_{2} \geq 0$, $k>1$ and $\lambda>0$ such that for almost $t \geq 0$ we have

$$
\phi^{\prime}(t)+K_{1} \phi(t)^{k} \leq K_{2}(1+t)^{-\lambda} .
$$

Then there exists a positive constant $M$ such that

$$
\phi(t) \leq M(1+t)^{-\nu}, \nu=\inf \left\{\frac{1}{k-1}, \frac{\lambda}{k}\right\} .
$$




\section{Proof of lemma 2.6}

Let $\lambda_{0}=\inf \left\{\lambda, \frac{k}{k-1}\right\}$, then we have for almost $t \geq 0$

$$
\phi^{\prime}(t)+K_{1} \phi(t)^{k} \leq K_{2}(1+t)^{-\lambda_{0}} .
$$

Let $\psi_{M}=M(1+t)^{-\frac{\lambda_{0}}{k}}=M(1+t)^{-\gamma}$. Then we get

$$
\psi_{M}^{\prime}(t)+K_{1} \psi_{M}(t)^{k}=K_{1} M^{k}(1+t)^{-\lambda_{0}}-\gamma M(1+t)^{-(1+\gamma)} .
$$

But we have $\lambda_{0} \leq 1+\gamma=1+\frac{\lambda_{0}}{k}$, it follows that

$$
\psi_{M}^{\prime}(t)+K_{1} \psi_{M}(t)^{k} \geq\left(K_{1} M^{k}-\gamma M\right)(1+t)^{-\lambda_{0}} .
$$

We choose $M$ such that $K_{1} M^{k}-\gamma M \geq K_{2}$ and $\psi_{M}(0)=M \geq \phi(0)$. Finally we have to use the maximum principle and then we obtain $\psi_{M} \geq \phi$.

\section{First order equations : Proof of Theorem 2.2}

It has been proved in [2] that there exists $\varphi \in \omega(u)$ such that

$$
\lim _{t \rightarrow \infty}\|u(t)-\varphi\|_{V}=0 \text {. }
$$

Let

$$
\Phi(t):=E(u(t))-E(\varphi)+\frac{1}{2} \int_{t}^{\infty}\|g(s)\|_{H}^{2} d s, \quad t \in \mathbb{R}_{+} .
$$

We have

$$
\begin{aligned}
\frac{d}{d t} \Phi(t) & =\langle\mathcal{M}(u(t)), \dot{u}(t)\rangle_{V^{\prime} \times V}-\frac{1}{2}\|g(t)\|_{H}^{2} \\
& =\langle\mathcal{M}(u(t)), \dot{u}(t)\rangle_{H \times H}-\frac{1}{2}\|g(t)\|_{H}^{2} \\
& =-\frac{1}{2}\left(\|\dot{u}(t)\|_{H}^{2}+\|\mathcal{M}(u(t))\|_{H}^{2}\right) .
\end{aligned}
$$

Hence, the function $\Phi$ is non increasing and $\lim _{t \rightarrow \infty} \Phi(t)=0$ which implies that $\Phi(t) \geq 0$ for all $t \in \mathbb{R}_{+}$.

We have

$$
\frac{d}{d t} \Phi(t) \leq-\frac{1}{2}\|\mathcal{M}(u(t))\|_{H}^{2} \leq-\frac{1}{2 C_{1}^{2}}\|\mathcal{M}(u(t))\|_{V^{\prime}}^{2}, \text { where } C_{1} \text { is as in }(3) .
$$


On the other hand since $\lim _{t \rightarrow \infty}\|u(t)-\varphi\|_{V}=0$, then there exists $T>0$ such that for all $t \geq T$ $\|u(t)-\varphi\|_{V}<\sigma$, where $\sigma$ is as in (6). Then by assumption 3 of theorem 2.2 together with Cauchy-Schwarz inequality we get for all $t \in[T, \infty[$

$$
\begin{aligned}
\frac{d}{d t} \Phi(t) & \leq-C_{2}|E(u)-E(\varphi)|^{2(1-\theta)} \\
& =-C_{2}\left|\Phi(t)-\frac{1}{2} \int_{t}^{\infty}\|g(s)\|_{H}^{2} d s\right|^{2(1-\theta)} \\
& \leq-C_{3} \Phi(t)^{2(1-\theta)}+C_{4}\left(\int_{t}^{\infty}\|g(s)\|_{H}^{2} d s\right)^{2(1-\theta)} .
\end{aligned}
$$

Thanks to assumption (7) we obtain the next differential inequality for all $t \geq T$

$$
\frac{d}{d t} \Phi(t)+C_{3} \Phi(t)^{2(1-\theta)} \leq C_{4}(1+t)^{-2(1+\delta)(1-\theta)} .
$$

By applying lemma 2.6, we get for all $t \geq T$

$$
\Phi(t) \leq C_{5}(1+t)^{-\gamma}
$$

where $\gamma=\inf \left\{\frac{1}{1-2 \theta}, 1+\delta\right\}$.

Now since we have

and by using (9) we get for all $t \geq T$

$$
\frac{1}{2}\|\dot{u}(t)\|_{H}^{2} \leq-\frac{d}{d t} \Phi(t)
$$

$$
\int_{t}^{2 t}\|\dot{u}(s)\|_{H}^{2} d s \leq 2 \Phi(t) \leq 2 C_{5}(1+t)^{-\gamma}
$$

Since we have $\int_{t}^{2 t}\|\dot{u}(s)\|_{H} d s \leq \sqrt{t}\left(\int_{t}^{2 t}\|\dot{u}(s)\|_{H}^{2} d s\right)^{\frac{1}{2}}$, we obtain for all $t \geq T$

$$
\int_{t}^{2 t}\|\dot{u}(s)\|_{H} d s \leq C_{6}(1+t)^{\frac{1-\delta}{2}} .
$$

It follows for all $t \geq T$

$$
\begin{aligned}
\int_{t}^{\infty}\|\dot{u}(s)\|_{H} d s & \leq \sum_{k=0}^{\infty} \int_{2^{k} t}^{2^{k+1} t}\|\dot{u}(s)\|_{H} d s \\
& \leq C_{7} \sum_{k=0}^{\infty}\left(2^{k} t\right)^{\frac{1-\gamma}{2}} \\
& \leq C_{8}(1+t)^{\frac{1-\gamma}{2}} .
\end{aligned}
$$

Finally we get for all $t \geq T$

$$
\|u(t)-\varphi\|_{H} \leq C(1+t)^{-\beta}
$$

where $\beta=\inf \left\{\frac{\theta}{1-2 \theta}, \frac{\delta}{2}\right\}$ and $C$ is a positive constant. 


\section{Second order equations : Proof of Theorem 2.3}

It has been proved in [2] that there exists $\varphi \in \omega(u)$ such that

$$
\lim _{t \rightarrow \infty}\left\{\|\dot{u}(t)\|_{H}+\|u(t)-\varphi\|_{V}\right\}=0 .
$$

Let

$$
\begin{aligned}
\Phi(t)= & \frac{1}{2}\|\dot{u}(t)\|_{H}^{2}+(E(u(t))-E(\varphi))+\varepsilon\langle\mathcal{M}(u(t)), \dot{u}(t)\rangle_{V^{\prime} \times V^{\prime}} \\
& +\int_{t}^{\infty}\langle g(s), \dot{u}(s)\rangle_{H \times H} d s+\varepsilon C_{1}^{2} \int_{t}^{\infty}\|g(s)\|_{H}^{2} d s,
\end{aligned}
$$

where $C_{1}$ is as in (3) and $\varepsilon$ is a real positive number which will be fixed in the sequel. We have

$$
\begin{aligned}
\frac{d}{d t} \Phi(t)= & \langle\dot{u}(t), \ddot{u}(t)\rangle_{H \times H}+\langle\mathcal{M}(u(t)), \dot{u}(t)\rangle_{V^{\prime} \times V}+\varepsilon\left\langle\mathcal{M}^{\prime}(u(t)) \dot{u}(t), \dot{u}(t)\right\rangle_{V^{\prime} \times V^{\prime}} \\
& +\varepsilon\langle\mathcal{M}(u(t)), \ddot{u}(t)\rangle_{V^{\prime} \times V^{\prime}}-\langle g(t), \dot{u}(t)\rangle_{H \times H}-\varepsilon C_{1}^{2}\|g(t)\|_{H}^{2} .
\end{aligned}
$$

Then we get

$$
\begin{gathered}
\frac{d}{d t} \Phi(t)=-\|\dot{u}(t)\|_{H}^{2}-\varepsilon\|\mathcal{M}(u(t))\|_{V^{\prime}}^{2}+\varepsilon\left\langle\mathcal{M}^{\prime}(u(t)) \dot{u}(t), \dot{u}(t)\right\rangle_{V^{\prime} \times V^{\prime}} \\
-\varepsilon\langle\mathcal{M}(u(t)), \dot{u}(t)\rangle_{V^{\prime} \times V^{\prime}}+\varepsilon\langle\mathcal{M}(u(t)), g(t)\rangle_{V^{\prime} \times V^{\prime}}-\varepsilon C_{1}^{2}\|g(t)\|_{H^{2}}^{2} .
\end{gathered}
$$

Thanks to Cauchy-Schwarz inequality we have

$$
\varepsilon\langle\mathcal{M}(u(t)), g(t)\rangle_{V^{\prime} \times V^{\prime}} \leq \frac{\varepsilon}{4}\|\mathcal{M}(u(t))\|_{V^{\prime}}^{2}+\varepsilon C_{1}^{2}\|g(t)\|_{H}^{2},
$$

where $C_{1}$ is as in (3).

Once again by applying Cauchy-Schwarz inequality and by assumption 4 of theorem 2.3 we obtain

$$
\begin{gathered}
\frac{d}{d t} \Phi(t) \leq-\|\dot{u}(t)\|_{H}^{2}-\varepsilon\|\mathcal{M}(u(t))\|_{V^{\prime}}^{2}+\frac{\varepsilon}{2}\left(C_{1}^{2}\|\dot{u}(t)\|_{H}^{2}+\|\mathcal{M}(u(t))\|_{V^{\prime}}^{2}\right) \\
+\varepsilon\left\|K \circ \mathcal{M}^{\prime}(u(t))\right\|_{\mathcal{L}(H)}\|\dot{u}(t)\|_{H}^{2}+\frac{\varepsilon}{4}\|\mathcal{M}(u(t))\|_{V^{\prime}}^{2}+\varepsilon C_{1}^{2}\|g(t)\|_{H}^{2}-\varepsilon C_{1}^{2}\|g(t)\|_{H}^{2} .
\end{gathered}
$$

We get

$$
\frac{d}{d t} \Phi(t) \leq-\left(1-\frac{\varepsilon}{2} C_{1}^{2}-\varepsilon\left\|K \circ \mathcal{M}^{\prime}(u(t))\right\|_{\mathcal{L}(H)}\right)\|\dot{u}(t)\|_{H}^{2}-\frac{\varepsilon}{4}\|\mathcal{M}(u(t))\|_{V^{\prime}}^{2}
$$

Choosing $\varepsilon>0$ small enough, we get for all $t \in \mathbb{R}_{+}$,

$$
\frac{d}{d t} \Phi(t) \leq-\frac{1}{2}\|\dot{u}(t)\|_{H}^{2}-\frac{\varepsilon}{4}\|\mathcal{M}(u(t))\|_{V^{\prime}}^{2} .
$$


Then the function $\Phi$ is noncreasing and $\lim _{t \rightarrow \infty} \Phi(t)=0$ which implies that $\Phi(t) \geq 0$ for all $t \in \mathbb{R}_{+}$.

By Cauchy-Schwarz inequality we get

$$
\begin{aligned}
& \Phi(t)^{2(1-\theta)} \leq 2|E(u)-E(\varphi)|^{2(1-\theta)}+2\left(\frac{1}{2}\|\dot{u}(t)\|_{H}^{2}+\varepsilon\left|\langle\mathcal{M}(u(t)), \dot{u}(t)\rangle_{V^{\prime} \times V^{\prime}}\right|+\right. \\
& \left.+\int_{t}^{\infty}\left|\langle g(s), \dot{u}(s)\rangle_{H \times H}\right| d s+\varepsilon C_{1}^{2} \int_{t}^{\infty}\|g(s)\|_{H}^{2} d s\right)^{2(1-\theta)}
\end{aligned}
$$

On the other hand since $\lim _{t \rightarrow \infty}\left\{\|\dot{u}(t)\|_{H}+\|u(t)-\varphi\|_{V}\right\}=0$, then there exists $T>0$ such that for all $t \geq T\|\dot{u}(t)\|_{H} \leq 1$ and $\|u(t)-\varphi\|_{V}<\sigma$, where $\sigma$ is as in (6).

Then thanks to assumption 5 of theorem 2.3 we obtain

$$
\begin{aligned}
& \Phi(t)^{2(1-\theta)} \leq 2 \eta^{2}\|\mathcal{M}(u(t))\|_{V^{\prime}}^{2}+2\left(\frac{1}{2}\|\dot{u}(t)\|_{H}^{2}+\varepsilon\left|\langle\mathcal{M}(u(t)), \dot{u}(t)\rangle_{V^{\prime} \times V^{\prime}}\right|+\right. \\
& \left.+\int_{t}^{\infty}\left|\langle g(s), \dot{u}(s)\rangle_{H \times H}\right| d s+\varepsilon C_{1}^{2} \int_{t}^{\infty}\|g(s)\|_{H}^{2} d s\right)^{2(1-\theta)},
\end{aligned}
$$

where $\eta$ is as in (6).

Now by using (10) together with Cauchy-Schwarz inequality, we get for all $t \in[T, \infty[$

$$
\begin{aligned}
\Phi(t)^{2(1-\theta)} \leq-C_{9} & \frac{d}{d t} \Phi(t)+C_{10}\left(\|\dot{u}(t)\|_{H}^{4(1-\theta)}+\|\mathcal{M}(u(t))\|_{V^{\prime}}^{2(1-\theta)}\|\dot{u}(t)\|_{V^{\prime}}^{2(1-\theta)}+\right. \\
& \left.+\left(\int_{t}^{\infty}\left|\langle g(s), \dot{u}(s)\rangle_{H \times H}\right| d s\right)^{2(1-\theta)}+\left(\int_{t}^{\infty}\|g(s)\|_{H}^{2} d s\right)^{2(1-\theta)}\right) .
\end{aligned}
$$

By using Young's inequality we obtain for all $t \geq T$

$$
\begin{array}{r}
C_{9} \frac{d}{d t} \Phi(t) \leq-\Phi(t)^{2(1-\theta)}+C_{10}\left(\|\dot{u}(t)\|_{H}^{4(1-\theta)}+\|\dot{u}(t)\|_{V^{\prime}}^{2 \frac{(1-\theta)}{\theta}}+\|\mathcal{M}(u(t))\|_{V^{\prime}}^{2}+\right. \\
\left.+\left(\int_{t}^{\infty}\left|\langle g(s), \dot{u}(s)\rangle_{H \times H}\right| d s\right)^{2(1-\theta)}+\left(\int_{t}^{\infty}\|g(s)\|_{H}^{2} d s\right)^{2(1-\theta)}\right) .
\end{array}
$$

On the other hand, thanks to Hölder's and Young's inequalities and by using (7) we have

$$
\left(\int_{t}^{\infty}\left|\langle g(s), \dot{u}(s)\rangle_{H \times H}\right| d s\right)^{2(1-\theta)} \leq \frac{1}{\nu} \frac{c^{2(1-\theta)}}{(1+t)^{2(1+\delta)(1-\theta)}}+\frac{\nu}{4}\left(\int_{t}^{\infty}\|\dot{u}(s)\|_{H}^{2} d s\right)^{2(1-\theta)}
$$

where $\nu$ is a small constant which will be fixed in the sequel.

By using (10), we have for all $t \geq T$

$$
\frac{d}{d t} \Phi(t) \leq-\frac{1}{2}\|\dot{u}(t)\|_{H}^{2}
$$


Then we get

$$
\left(\int_{t}^{\infty}\|\dot{u}(s)\|_{H}^{2} d s\right)^{2(1-\theta)} \leq 2^{2(1-\theta)}(\Phi(t))^{2(1-\theta)} .
$$

Now since we have for all $t \geq T\|\dot{u}(t)\|_{H} \leq 1$, by using the fact that $4(1-\theta) \geq 2,2\left(\frac{1-\theta}{\theta}\right) \geq$ 2 , together with (11) and by choosing $\nu$ such that $\nu \leq \frac{2^{2 \theta-1}}{C_{10}}$, we get for all $t \geq T$

$$
C_{9} \frac{d}{d t} \Phi(t) \leq-\frac{1}{2} \Phi(t)^{2(1-\theta)}+C_{11}\left(\|\dot{u}(t)\|_{H}^{2}+\|\mathcal{M}(u(t))\|_{V^{\prime}}^{2}+(1+t)^{-2(1-\theta)(1+\delta)}\right) .
$$

Now thanks to (10) and (12) we get the following differential inequality for all $t \geq T$

$$
C_{12} \frac{d}{d t} \Phi(t)+\frac{1}{2} \Phi(t)^{2(1-\theta)} \leq C_{11}(1+t)^{-2(1-\theta)(1+\delta)} .
$$

Then we are able to apply lemma 2.6, and so we obtain

$$
\Phi(t) \leq C_{13}(1+t)^{-\gamma}
$$

where $\gamma=\inf \left\{\frac{1}{1-2 \theta}, 1+\delta\right\}$.

Thanks to (10) we have

$$
\|\dot{u}(t)\|_{H}^{2} \leq-2 \frac{d}{d t} \Phi(t)
$$

and then by integrating over $[t, 2 t]$ and by using (13) we get for all $t \geq T$

$$
\int_{t}^{2 t}\|\dot{u}(s)\|_{H}^{2} d s \leq C_{14}(1+t)^{-\gamma} .
$$

Since we have

$$
\int_{t}^{2 t}\|\dot{u}(s)\|_{H} d s \leq \sqrt{t}\left(\int_{t}^{2 t}\|\dot{u}(s)\|_{H}^{2} d s\right)^{\frac{1}{2}} .
$$

It follows that

$$
\int_{t}^{2 t}\|\dot{u}(s)\|_{H} d s \leq C_{15}(1+t)^{\frac{1-\gamma}{2}}
$$

Therefore we get for all $t \geq T$

$$
\begin{aligned}
\int_{t}^{\infty}\|\dot{u}(s)\|_{H} d s & \leq \sum_{k=0}^{\infty} \int_{2^{k} t}^{2^{k+1} t}\|\dot{u}(s)\|_{H} d s \\
& \leq C_{16} \sum_{k=0}^{\infty}\left(2^{k} t\right)^{\frac{1-\gamma}{2}} \\
& \leq C_{17}(1+t)^{\frac{1-\gamma}{2}}
\end{aligned}
$$


Finally we obtain for all $t \geq T$

$$
\|u(t)-\varphi\|_{H} \leq C^{\prime}(1+t)^{-\beta}
$$

where $\beta$ is as in (8) and $C^{\prime}$ is a positive constant.

\section{Applications}

The object of this section is to specify the rate of decay to equilibrium for the list of examples which were considered in [2].

\subsection{Systems of ordinary differential equations}

Let $F: \mathbb{R}^{N} \rightarrow \mathbb{R}$ be of class $C^{2}$, and let $g \in L_{l o c}^{1}\left(\mathbb{R}_{+} ; \mathbb{R}^{N}\right)$. Then the theorems 2.2 and 2.3 apply to the systems of ordinary differential equations

$$
\begin{cases}\dot{u}(t)+\nabla F(u(t))=g(t), & t \geq 0, \\ u(0)=u_{0}, & u_{0} \in \mathbb{R}^{N},\end{cases}
$$

and

$$
\begin{cases}\ddot{u}(t)+\dot{u}(t)+\nabla F(u(t))=g(t), & t \geq 0, \\ u(0)=u_{0}, \dot{u}(0)=u_{1}, & u_{0}, u_{1} \in \mathbb{R}^{N} .\end{cases}
$$

It suffices to choose $V=H=\mathbb{R}^{N}$ and $\mathcal{M}(u(t))=\nabla F(u(t))$. In this case, obviously, every bounded solution $u$ has precompact range. The regularity of $u$ follows from the regularity of $F$. Thus, the main assumption which has to be checked is assumption 3 in theorem 2.2, resp. assumption 5 in theorem 2.3. For these, we have the following result.

Proposition 5.1 The function $F$ satisfies the Lojasiewicz-Simon inequality near some $\varphi \in$ $\mathbb{R}^{N}$ if one of the following two cases (i) or (ii) holds:

(i) the function $F$ is analytic in a neighbourhood of $\varphi$, or

(ii) the connected component $C$ of the set $\left\{z \in \mathbb{R}^{N}: \nabla F(z)=0\right\}$ which contains $\varphi$ has locally near $\varphi$ the same dimension as the kernel of $\nabla^{2} F(\varphi)$, and $\varphi$ lies in the interior of the component $C$.

Remark 5.2 Assumption (i) of proposition 5.1 is just the Lojasiewicz inequality and assumption (ii) is proved in [16] (see also [7]).

Our main results are the following and follow from theorems 2.2 and 2.3: 
Corollary 5.3 Let $u \in W^{1, \infty}\left(\mathbb{R}^{+}, \mathbb{R}^{N}\right)$ be a solution of (14). We assume that $F$ verifies one of assumptions (i),(ii) of proposition 5.1. Then there exists a constant $C>0$ such that for all $t \geq 0$ we have

$$
\|u(t)-\varphi\| \leq C(1+t)^{-\beta}
$$

where $\beta$ is as in (8).

Corollary 5.4 Let $u \in W^{1, \infty}\left(\mathbb{R}^{+}, \mathbb{R}^{N}\right)$ be a solution of (15). We assume that $F$ verifies one of assumptions (i),(ii) of proposition 5.1. Then there exists a constant $C^{\prime}>0$ such that for all $t \geq 0$ we have

$$
\|u(t)-\varphi\| \leq C^{\prime}(1+t)^{-\beta}
$$

where $\beta$ is as in (8).

\subsection{Heat equation}

As a next application we study the asymptotic behavior of the semilinear heat equation

$$
\begin{cases}u_{t}-\Delta u+f(x, u)=g(t, x), & (t, x) \in \mathbb{R}_{+} \times \Omega, \\ \left.u(t, \cdot)\right|_{\partial \Omega}=0, & t \in \mathbb{R}_{+}, \\ u(0, x)=u_{0}(x), & x \in \Omega .\end{cases}
$$

In equation (16) we assume that $\Omega \subset \mathbb{R}^{d}(d \geq 1)$ is a bounded domain. We assume that the function $f: \bar{\Omega} \times \mathbb{R} \rightarrow \mathbb{R}$ is continuously differentiable and if $d \geq 2$ then we assume in addition that

$$
\begin{aligned}
& \text { there exists }\left\{\begin{array}{ll}
0 \leq \alpha \leq \frac{2}{d-2} & \text { if } d \geq 2 \\
0 \leq \alpha<\infty & \text { if } d=2
\end{array} \quad\right. \text { such that } \\
& \sup _{x \in \Omega, s \in \mathbb{R}}\left(1+|s|^{\alpha}\right)^{-1}\left|\frac{\partial}{\partial s} f(x, s)\right|<\infty .
\end{aligned}
$$

We assume in addition $g \in L_{\text {loc }}^{2}\left(\mathbb{R}_{+} ; L^{2}(\Omega)\right)$, and we will rewrite the equation (16) in an abstract setting, i.e. on the Hilbert space $H:=L^{2}(\Omega)$. We let $(A, D(A))$ be the DirichletLaplace operator defined by

$$
\begin{aligned}
D(A) & :=\left\{u \in H_{0}^{1}(\Omega): \exists v \in L^{2}(\Omega) \forall \varphi \in H_{0}^{1}(\Omega) \int_{\Omega} \nabla u \nabla \varphi d x=\int_{\Omega} v \varphi d x\right\}, \\
A u & :=v .
\end{aligned}
$$

We let, moreover, $V:=H_{0}^{1}(\Omega)$ and $\tilde{f}: V \rightarrow H$ the Nemytskii operator associated with $f$, i.e.

$$
\tilde{f}(v)(x):=f(x, v(x)), \quad v \in V, x \in \Omega .
$$

Note that, by the inequality (17) and by the Sobolev embeddings, the operator $\tilde{f}$ is Lipschitz continuous from bounded subsets of $V$ with values in $H$, and continuously differentiable from $V$ with values in $V^{\prime}$. 
With this notation, equation (16) becomes the abstract Cauchy problem

$$
\begin{cases}\dot{u}+A u+\tilde{f}(u)=g(t), & t \in \mathbb{R}_{+}, \\ u(0)=u_{0}, & u_{0} \in H,\end{cases}
$$

which is in fact a special case of equation (4) if we define the energy functional $E: V \rightarrow \mathbb{R}$ by

$$
E(u):=\frac{1}{2} a(u, u)+\int_{\Omega} F(x, u) d x, \quad u \in V .
$$

Here, $F(x, s):=\int_{0}^{s} f(x, r) d r$, and $a: V \times V \rightarrow \mathbb{R}$ is the bilinear form associated with the Dirichlet-Laplace operator, i.e.

$$
a(u, v):=\int_{\Omega} \nabla u \nabla v d x, \quad u, v \in V .
$$

With this definition of $E$ we have $\mathcal{M} u=A u+\tilde{f}(u)$ for every $u \in V$.

R. Chill and M.A. Jendoubi have showed in [2] that conditions of theorem 2.2 are verified in this case and that if $E$ verifies one of the assumptions of the next proposition, for every bounded solution $u: \mathbb{R}_{+} \rightarrow H$ of equation (18) we have $\lim _{t \rightarrow \infty} u(t)=\varphi$ in $V$.

Proposition 5.5 Let $E$ be the energy functional defined in equation (19), assume $E \in$ $C^{2}(V ; \mathbb{R})$, and denote by $\mathcal{M}^{\prime}$ the second derivative of $E$. Then $E$ satisfies the LojasiewiczSimon inequality near some $\varphi \in V$ if one of the following two cases (i) or (ii) holds:

(i) $\operatorname{Ker} \mathcal{M}^{\prime}(\varphi) \subset L^{\infty}(\Omega)$, and the function $f$ is analytic in the second variable, uniformly in $x \in \Omega$, or

(ii) the connected component $C$ of the set $\{v \in V: \mathcal{M}(v)=0\}$ which contains $\varphi$ has locally near $\varphi$ the same dimension as the kernel of $\mathcal{M}^{\prime}(\varphi)$, and $\varphi$ lies in the interior of the component $C$.

Remark 5.6 Assumption (i) of proposition 5.5 is just the Lojasiewicz-Simon inequality and assumption (ii) is proved in [16](see also [7]).

The following result is an immediate application of theorem 2.2 using the convergence result established by R. Chill and M.A. Jendoubi.

Theorem 5.7 Let $u: \mathbb{R}_{+} \rightarrow H$ be a bounded solution of equation (18). Let $\varphi \in \omega(u)$, and assume that one of the two cases (i) or (ii) of proposition 5.5 is satisfied. Assume that $g$ satisfies the assumption 4 of theorem 2.2. Then there exists a constant $C>0$ such that for all $t \geq 0$ we have

$$
\|u(t)-\varphi\|_{H} \leq C(1+t)^{-\beta}
$$

where $\beta$ is as in (8). 


\subsection{Wave equations}

As a next application we study the asymptotic behavior of the semilinear wave equation

$$
\begin{cases}u_{t t}+u_{t}-\Delta u+f(x, u)=g(t, x), & (t, x) \in \mathbb{R}_{+} \times \Omega, \\ \left.u(t, \cdot)\right|_{\partial \Omega}=0, & t \in \mathbb{R}_{+}, \\ u(0, x)=u_{0}(x), u_{t}(0, x)=u_{1}(x), & x \in \Omega\end{cases}
$$

We let $\Omega \subset \mathbb{R}^{d}, f \in C^{1}(\bar{\Omega} \times \mathbb{R} ; \mathbb{R}), g \in L_{\text {loc }}^{1}\left(\mathbb{R}_{+} ; L^{2}(\Omega)\right)$, the spaces $H:=L^{2}(\Omega)$ and $V:=H_{0}^{1}(\Omega)$, the Dirichlet-Laplace operator $A$, and the Nemytskii operator $\tilde{f}$ as in Subsection 5.2 . If $d \geq 2$, then we replace the growth condition (17) by the following condition:

$$
\begin{aligned}
& \text { there exists } 0 \leq \alpha<\frac{2}{d-2} \text { such that } \\
& \sup _{s \in \mathbb{R}, x \in \Omega}\left(1+|s|^{\alpha}\right)^{-1}\left|\frac{\partial}{\partial s} f(x, s)\right|<\infty .
\end{aligned}
$$

With this notation, equation (20) can be abstractly rewritten on the space $V^{\prime}$ :

$$
\begin{cases}\ddot{u}+\dot{u}+A u+\tilde{f}(u)=g(t), & t \in \mathbb{R}_{+}, \\ u(0)=u_{0}, & u_{0} \in V, \\ \dot{u}(0)=u_{1}, & u_{1} \in H\end{cases}
$$

This equation is a special case of equation (5) if we define the energy functional $E: V \rightarrow \mathbb{R}$ as in equation (19). Note that $\mathcal{M} u=A u+\tilde{f}(u)$ for every $u \in V$.

Since the energy $E$ is the energy defined in (19), proposition 5.5 applies also in the case of the wave equation.

As for the heat equation, R. Chill and M.A. Jendoubi established that conditions of theorem 2.3 are verified. They proved thanks to proposition 5.5 that for every solution $u \in W_{\text {loc }}^{1,2}\left(\mathbb{R}_{+} ; V\right) \cap W_{\text {loc }}^{2,2}\left(\mathbb{R}_{+} ; H\right)$ of equation (22) such that $\{(u(t), \dot{u}(t)): t \geq 0\}$ is bounded in $V \times H$, we have $\lim _{t \rightarrow \infty} u(t)=\varphi$ in $V$.

The following result is an immediate application of theorem 2.3 using the convergence result established by R. Chill and M.A. Jendoubi.

Theorem 5.8 Let $u \in W_{\text {loc }}^{1,2}\left(\mathbb{R}_{+} ; V\right) \cap W_{\text {loc }}^{2,2}\left(\mathbb{R}_{+} ; H\right)$ be a solution of equation (22) such that $\{(u(t), \dot{u}(t)): t \geq 0\}$ is bounded in $V \times H$. Let $\varphi \in \omega(u)$, and assume that the case (i) or the case (ii) of proposition 5.5 holds. Suppose that $g$ satisfies assumption 6 of theorem 2.3. Then there exists a constant $C^{\prime}$ such that for all $t \geq 0$ we have

$$
\|u(t)-\varphi\|_{H} \leq C^{\prime}(1+t)^{-\beta}
$$

where $\beta$ is as in (8).

Acknowledgments. The author would like to express her sincere gratitude to Professor A. Haraux for stimulating discussions. The author wishes also to thank warmly Professor M.A. Jendoubi for his guidance. 


\section{References}

[1] P. Brunovský and P. Polačik, On the local structure of $\omega$-limit sets of maps, Z. Angew. Math. Phys 48 (1997), 976-986.

[2] R. Chill and M.A. Jendoubi, Convergence to steady states in asymptotically autonomous semilinear evolution equations, Nonlinear Analysis 53 (2003), 1017-1039.

[3] J. K. Hale and G. Raugel, Convergence in gradient-like systems with applications to PDE, Z. Angew. Math. Phys. 43 (1992), 63-124.

[4] A. Haraux, Slow and fast decay of solutions to some second order evolution equations, Journal d'analyse mathématique, 95 (2005), 297-321.

[5] A. Haraux and M.A. Jendoubi, Decay estimates to equilibrium for some evolution equations with an analytic nonlinearity, Asymptotic Analysis 26 (2001), 21-36.

[6] A. Haraux and M. A. Jendoubi, Convergence of bounded weak solutions of the wave equation with dissipation and analytic nonlinearity, Calc. Var. Partial Differential Equations, 9 (1999), 95-124.

[7] A. Haraux and M.A. Jendoubi, On the convergence of global and bounded solutions of some evolution equations, J Evol. Equ. 7 (2007), no.3, 449-470.

[8] A. Haraux and P. Polačik, Convergence to a positive equilibrium for some nonlinear evolution equations, Acta Math. Univ. Comen. 61 (1992), 129-141.

[9] S.-Z. Huang and P. Takač, Convergence in gradient-like systems which are asymptotically autonomous and analytic, Nonlinear Analysis 46 (2001), 675-698.

[10] M. A. Jendoubi, Convergence of global and bounded solutions of the wave equation with linear dissipation and analytic nonlinearity, J Diff. Equ. 144 (1998), 302-312.

[11] _ A simple unified approach to some convergence theorems of L. Simon, J. Funct. Anal. 153 (1998), 187-202.

[12] P.-L. Lions, Structure of the set of steady-state solutions and asymptotic behaviour of semilinear heat equations, J Diff. Equ. 53 (1984), 362-386.

[13] S. Lojasiewicz, Ensembles semi-analytiques, Preprint, I.H.E.S. Bures-sur-Yvette, 1965.

[14] H. Matano, Convergence of solutions of one-dimensional semilinear heat equations, J. Math. Kyoto Univ. 18 (1978), 221-227.

[15] L. Simon, Asymptotics for a class of non-linear evolution equations, with applications to geometric problems, Ann. of Math. 118 (1983), 525-571. 
[16] Theorems on Regularity and Singularity of Energy Minimizing Maps, Lecture Notes in Mathematics ETH Zürich, Birkhäuser Verlag, Basel, 1996.

[17] T. I. Zelenyak, Stabilization of solutions of boundary value problems for a second-order parabolic equation with one space variable, Differ. Eq. 4 (1968), 17-22, Transl. from Differ. Uravn. 4 (1968), 34-45. 